\title{
Erratum: Realization of a heavily doped and fully compensated semiconductor state in a crystalline semiconductor with a deep impurity band [JETP 84, 309-316 (February 1997)]
}

M. I. Daunov, I. K. Kamilov, and A. B. Magomedov

Institute of Physics, Dagestan Science Center, Russian Academy of Sciencees, 367003 Makhachkala, Russia

[S1063-7761(97)02406-2]

The following corrections were reported by the authors. On page 309 in the right-hand column, in the 10th line from the bottom, the equation should read as follows: $N_{d}{ }^{+}=N_{d \mu}{ }^{+}+N_{d r}{ }^{+}$. On the same page and the same column, in the 9th line from the bottom, the equation should read as follows: $N_{a}=N_{a r}{ }^{-}+N_{a \mu}{ }^{-}+N_{A}$. Also on the same page and the same column, in the 2nd line from the bottom, the equation should read as follows: $N=N_{d}{ }^{+}-\left(N_{a \mu}{ }^{-}+N_{a r}{ }^{-}\right)$.

In the first part of Table II, in the 11th column, in the second line from the bottom, the number should read as follows: 0.02 . 